\title{
Introducing Time-Delays to Analyse Driver Reaction Times when Using a Powered Wheelchair
}

\author{
David Sanders ${ }^{1}$, Malik Haddad ${ }^{1}$, Martin Langner ${ }^{2}$, Peter Omoarebun ${ }^{1}$, \\ John Chiverton ${ }^{1}$, Mohamed Hassan ${ }^{3}$, Shikun Zhou ${ }^{1}$, Boriana Vatchova ${ }^{4}$ \\ ${ }^{1}$ Faculty of Technology, University of Portsmouth, Portsmouth PO1 3DJ, UK \\ ${ }^{2}$ Chailey Heritage Foundation, North Chailey, BN8 4E, UK \\ ${ }^{3}$ School of Chemical Engineering, University of Southampton, Southampton, SO17 1BJ, UK \\ ${ }^{4}$ Bulgarian Academy of Sciences, 1113 Sofia Akad.G.Bonchev str., bl.2, Bulgaria \\ david.sanderseport.ac.uk
}

\begin{abstract}
This paper investigates the introduction of time-delays into wheelchair driving. Two dissimilar ways in which wheelchair drivers interact are compared. Users were observed as they drove their wheelchairs with and without timedelays. Tests took place with a computer system and sensors which provided assistance and then without any assistance provided. As delays became longer then drivers found it more difficult to drive. If the wheelchair moved through a more complicated environment or if the time-delay was made longer, then driving was better if the computer and sensors assisted. Time delays were introduced between the motor controller and the wheelchair joystick. With shorter time-delays or in simpler environments then less assistance was needed from the computer system and sensors. In more complicated environments or if time-delays were longer, then more assistance was needed. That suggest varying sensor support could be helpful depending on the complexity of the environment or the difficulties being experienced by the drivers.
\end{abstract}

Keywords: Ultra-sonic, sensor, time-delay, wheelchair, intelligent.

\section{Introduction}

Time-delays are investigated in this paper and their effect on the performance of wheelchair drivers is discussed. Many things can affect reaction times (ReTis), for example: age, gender, personality type, tiredness, distraction, physical fitness, alcohol and whether stimuli are auditory or visual [1]. Specifically, Parkinson's sufferers have a longer ReTi [2]. Numerous dissimilar environments are considered. In each case, drivers used a joystick to control their chair and could use ultrasonic sensors to assist them. They completed a series of tasks with and without sensors to help them.

The next Section provides some background to give context to the work and then the sensors and wheelchair hardware are described. After that, the testing is presented. Finally the paper ends with some discussion, conclusions and future work. A significant deduction is that drivers perform better in simpler situations without sensors 
to assist [3-6]. Failures increased noticeably if the sensors weren't used or if timedelays were increased.

\section{Background}

Time delays can be created by slower reactions. Slower reactions add distortion to controller commands and feed-back [7] and that can reduce performance [8]. Some approaches to control with delays are described in $[9,10]$.

$\mathrm{ReTi}$ is defined in [1] as the time for a message to travel from a sensor (e.g.: eyes) to the brain and then to an actuator (e.g.: a muscle in your leg). Neurons transmit messages to and from the brain and spinal cord. Numerous issues affect ReTi [14] and slower ReTis can cause accidents during driving [14]. People suffering from akinesia have appreciably longer ReTis $[11,12]$ or a lesion in the right basal ganglia will cause ReTi to get longer [13,14].

The information flow can be represented as: Neuron Stimulus sent to Spinal-Cord - then - from Brain to Actuator to Neuron and on to Response. Sensor neurons convert stimuli to electro-chemical signals within sensory neurons. The signal journeys through the nervous system to the motor neurons. The motor neurons cause muscles to change shape or glands to secrete. Factors that affect ReTi are:

- Age. ReTi gets shorter until late twenties, then gets longer slowly until the 60s when ReTi gets longer more quickly.

- Left vs Right. ReTi in left-handed people are often faster.

- Practice. Following errors, ReTis get longer. Training shortens ReTi and improves accuracy.

- $\quad$ Errors. If a task is new, then ReTis are less consistent.

- Physical Tiredness. ReTi slows when tired.

- Mental Tiredness. Sleep deprivation or sleepiness lengthens ReTi and causes people to miss stimuli.

- Distraction. ReTi increases with distractions. Additionally, ReTis get longer when verbal tasks are being given.

- Warning. ReTis are quicker if a warning is given.

- Alcohol. Drink slows ReTi because muscle activation becomes slower.

- $\quad$ Finger Tremble. Fingers tremble and ReTis are quicker if a reaction is happening on a 'downswing'.

- Personality. Anxious personalities and extroverts have faster ReTis. Neurotics and Schizophrenics have slower ReTis.

- Exercise. Fitter people have quicker ReTis.

- Threats or Stress. Making a person anxious can make reactions faster.

- $\quad$ Stimulant. Caffeine makes ReTi faster and smokers refraining from smoking have faster ReTis. Some drugs make ReTi faster.

- Learning Disorders. People with language and reading difficulties tend ot have slower ReTis. 
- Injury to the brain. Brain injury can slow down ReTi and concussions and headaches can reduce performance.

- Illness. Minor upper respiratory tract infection can slow ReTi.

Work described here was mainly interested in time delays because of aging, illness, learning disorders and brain injury but also considered effects of errors, practice and fatigue.

Powered wheelchairs are often controlled with a joystick [15] but other transducers can be used: pointers [16,17], switches [18,19], or virtual reality headsets [20]. Controllers interface lower current inputs to higher current actuators that drive motors connected to wheels. Variances in wheels and different gradients and surfaces can cause veer [18,19] and time-delays [9, 21-23] can occur because of longer ReTis[14]. The drivers react to disturbances and correct wheelchair direction and speed.

\section{The System}

Ultrasonics are simple and robust [24] and in this work, $40 \mathrm{KHz}$ transmitter / receiver pairs were mounted at the front of the wheelchair to provide a basic ultrasound image of the environment.

A joystick controlled the electrical current to DC servo amplifiers and motors on a BobCat II wheelchair. A computer was inserted between the joystick and the servo amplifiers [25]. The ultrasonics sensed objects around the wheelchair and the computer modified the user control signals in the light of the ultrasonic image. The system is described in $[4,6,26,27]$. The computer controlled the wheelchair but considered input from the joystick and the sensors. The computer quizzed the sensors and adjusted wheelchair direction.

Joystick data could go straight to the wheelchair controller so that the wheelchair would react directly to input from the joystick. Software was assembled as described in [28] with three levels (Servo, Strategic and Supervisory [29, 30]).

These rules were applied: A trajectory was only adjusted if necessary; Movements were smooth and controlled; The driver remained in overall control.

\section{$4 \quad$ Trials}

Trials took place to:

- Compare systems when jointly controlled with a mix of human and computer control, with human control, when a variety of time-delays were introduced to represent various ReTis.

- Record the number of successful tasks and failures with different time-delays and in different conditions.

- Record any improvements achieved when using the system to assist, especially when time-delays were introduced. 
- Record the time taken to complete tasks with the sensors and without them as time-delays increased and gaps reduced.

- Record the smallest gap that human users could safely drive through as the timedelay was lengthened, both with the sensors and without them.

Wheelchair drivers quickly learnt how the systems worked and responded.

There were eight clusters of trials for each driving route. Four without any automatic assistance from the sensors and four with automatic assistance from the sensors. Obstacle courses were created for each trial in a variety of environments:

INSIDE LABORATORY: Two objects on a flat floor with upright walls.

INSIDE SIMPLE CORRIDOR: Upright walls with flat and some sloping surfaces with some objects. No doorways.

INSIDE COMPLICATED CORRIDOR: Doorways and upright walls, with flat and sloping surfaces. Some radiators and door surrounds. Numerous obstacles.

OUTSIDE: More complex environment with various flat and sloping surfaces and vertical edges. Various natural obstacles and objects.

A clear explanation was provided to participating volunteers that included risks and benefits. There were 15 female and 36 male participants. The 51 volunteers were 1851 years old (SD 4.8, Mean 22). Trials were repeated as human performance varied.

Drivers repeated trials and could learn and were able to perform at their best in the time available. As trails were repeated, then time-delays became longer. Volunteers tried to beat their best performances. The number of failed runs and successful runs were recorded. A successful run was collision free. A failure included a collision(s).

The initial set of trials used routes with objects set $90 \mathrm{~cm}$ apart $(10 \mathrm{~cm}$ wider than the powered wheelchair). Then trials were repeated with thinner gaps. If a trial was successful with a thinner gap then a driver made at least one more attempt at the other test (with or without the sensor system assisting them). If they were successful again then another attempt was made with the original setup. Trial routes started from a standing start at set starting position. Gaps were verified by two researchers using a ruler and measure.

Fig.1 shows where the delays were introduced (h2). The velocity command to the motors (v1) could be delayed so that signal (vr) was delayed. $\mathrm{h}$ was the total time-delay, that is $\mathrm{h} 2$ (the forward delay) and $\mathrm{h} 1$ (the backward delay).

Fig. 2 shows Indoor Complicated Corridor Three. Arrows show the route for the wheelchairs. Shaded blocks show the objects in the path of the powered wheelchairs. That route also had two double-doorways. There, one door was open and one kept shut. So a chair had to zig-zag to successfully pass them.

A camera on the chair observed and recorded trials. Fig. 3 shows a scene from the camera. The pictures show a successful trial run with a delay of $2.1 \mathrm{~s}$. 




Fig. 1. Delays in the system. Based on the system in [10].

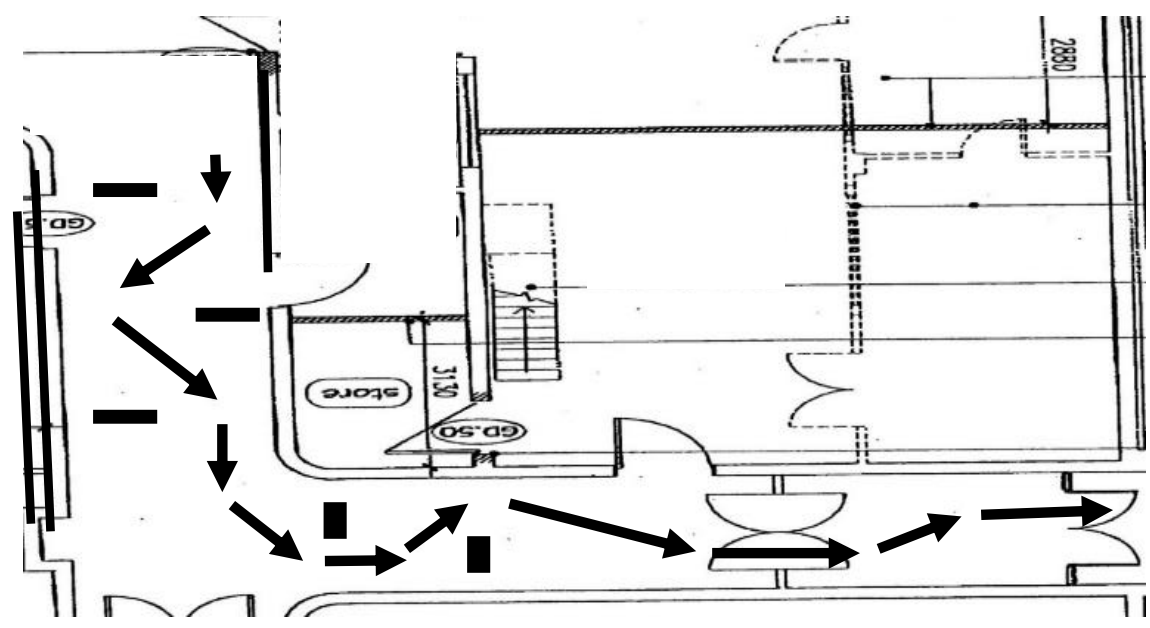

Fig. 2. Complicated Corridor One. 

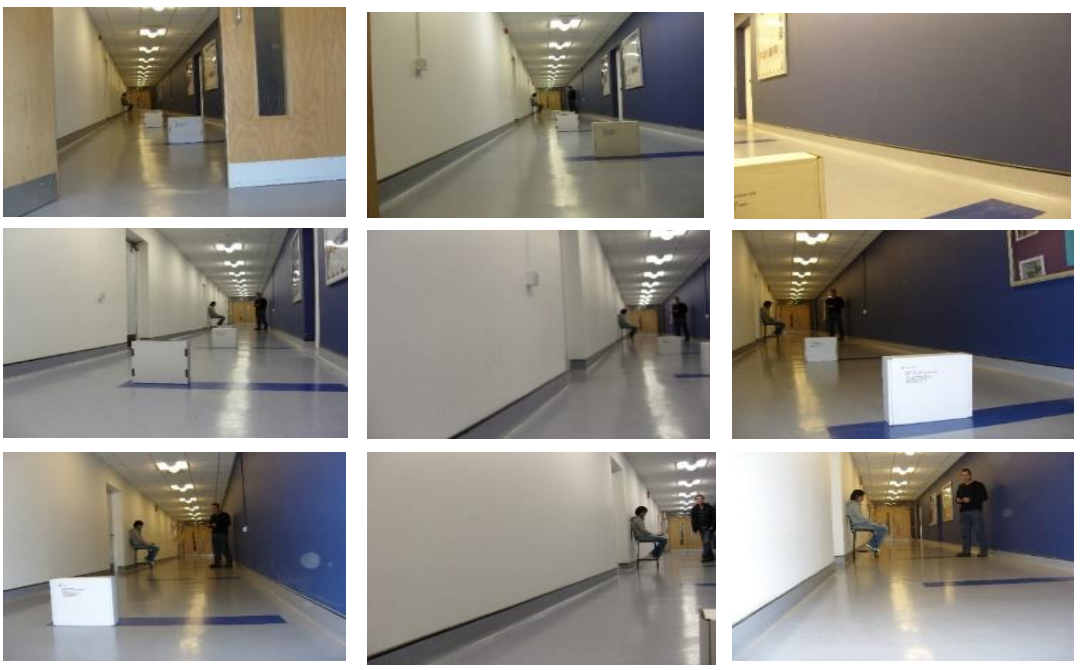

Fig. 3. Camera view when moving through an indoor complicated corridor.

\section{$5 \quad$ Results}

The wheelchair automatically avoided obstacles when the assistive computer systems were connected. There were some chaotic factors that affected the result, including variation in wheel position, slope, floor surface, or the trailing casters could send a chair off the desired path.

\subsection{Operation With and Without Sensors.}

Fig.4 displays the average of best time to finish a variety of routes. Average time to finish successful runs is shown on the vertical scale. Simple environments are to the left in each graph shown in the figures, for example empty corridors and laboratory. The results show that drivers completed the simpler routes more quickly when they did not have any assistance from the sensors and computer system. More complex routes are shown to the right, for example outside routes and complicated corridors.

Wheelchair users finished the more complex routes faster when the sensor and computer system were connected and working. The lower graph shows the average of fastest times when a 1s delay was introduced.

Each time a test took place, gaps were reduced by $0.5 \mathrm{~cm}$. The thinnest set of gaps that a driver successfully navigated through were recorded with the number of failed and successful runs. Drivers completed courses with thinner gaps when utilizing the computer and sensors. Fig. 5 shows the average improvement in $\mathrm{cm}$ when using the sensors and microcomputer. The graph at the top is without any time-delay and the graph at the bottom is with a 1s delay. As simpler environments were changed into more complex environments or gaps changed to be thinner then drivers found it trickier to judge the width between obstacles. It was more difficult for them to pass through the thinner gaps. Drivers relied more on the sensor and computer systems. 

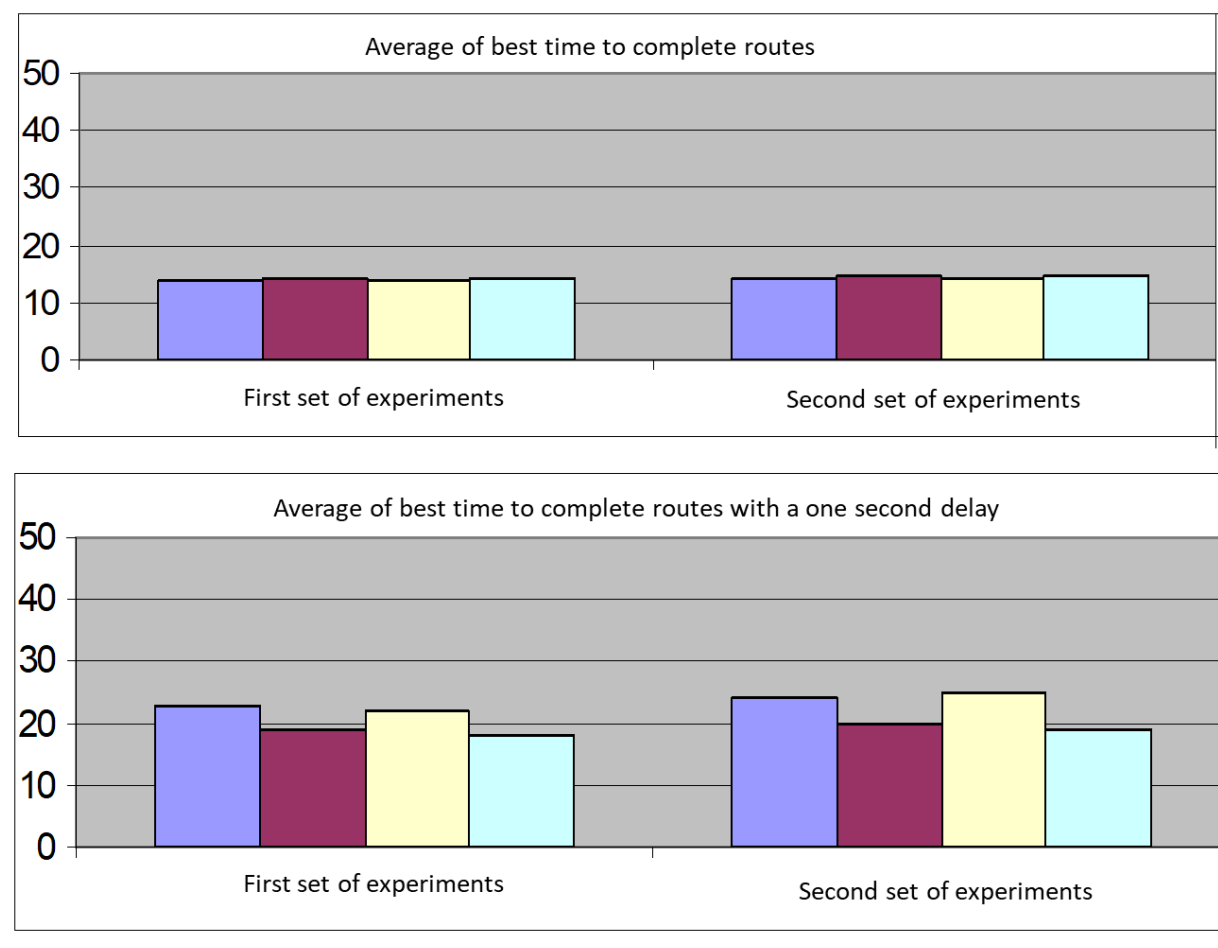

Fig. 4. Average of the best time taken to complete a route.

Drivers successfully drove through thinner gaps when the microcomputer and sensors were being used. Gradients, hills and surfaces had a tendency to turn the chairs and sensors became more useful in those cases. The microcomputer consistently corrected wheelchair angles and as time-delays increased then the results were more noticeable. Wheelchairs were driven faster through thinner gaps with the assistance from the sensors, especially when time-delays increased. Fig. 5 shows the gaps acheived with and without the sensors engaged and without any delay (top). The bottom graph shows the gaps achieved with and without the sensor systems engaged but with a delay of one second introduced. 


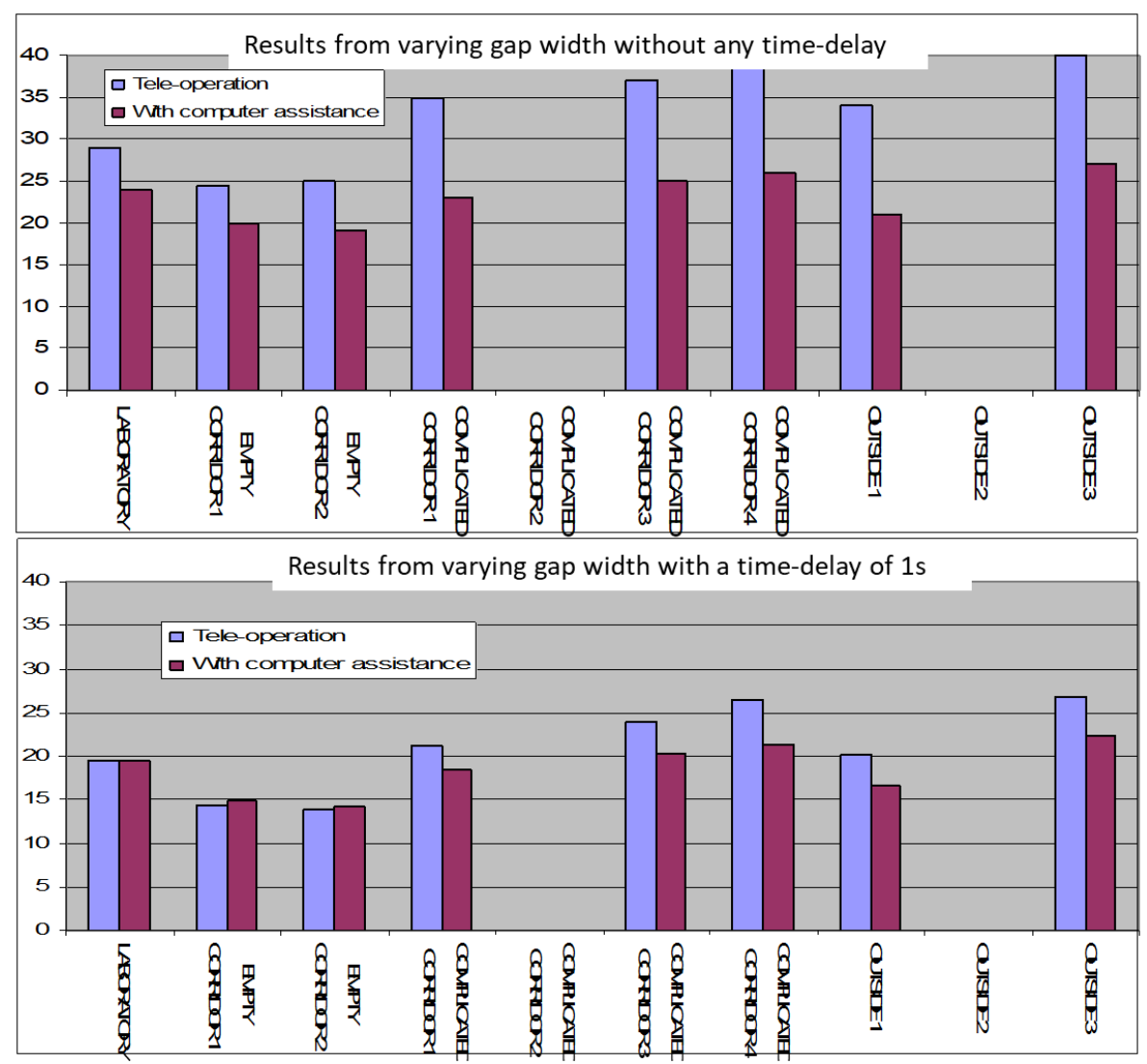

Fig. 5. Reducing gap widths with a time-delay of $1.5 \mathrm{~s}$ (bottom).

\subsection{Times to complete courses}

As gaps became thinner and time-delays increased, then the fastest time to complete routes and tasks was logged. When gaps widened, drivers completed courses faster without sensors and when gaps became thinner then wheelchair drivers completed routes and tasks faster with sensors and the microcomputer.

\subsection{Failure Rates}

Fig. 6 displays failed and successful attempts with and without sensors and the microcomputer assisting drivers. The $\mathrm{x}$ axis is a list of different environments. Average numbers of failed and successful trials is to the left. The center bar shows percentage of failed attempts. The right shows the difference between failures when being assisted and when not. The top bar chart is when there was not any delay and at the bottom are the results with 1 s delays. 

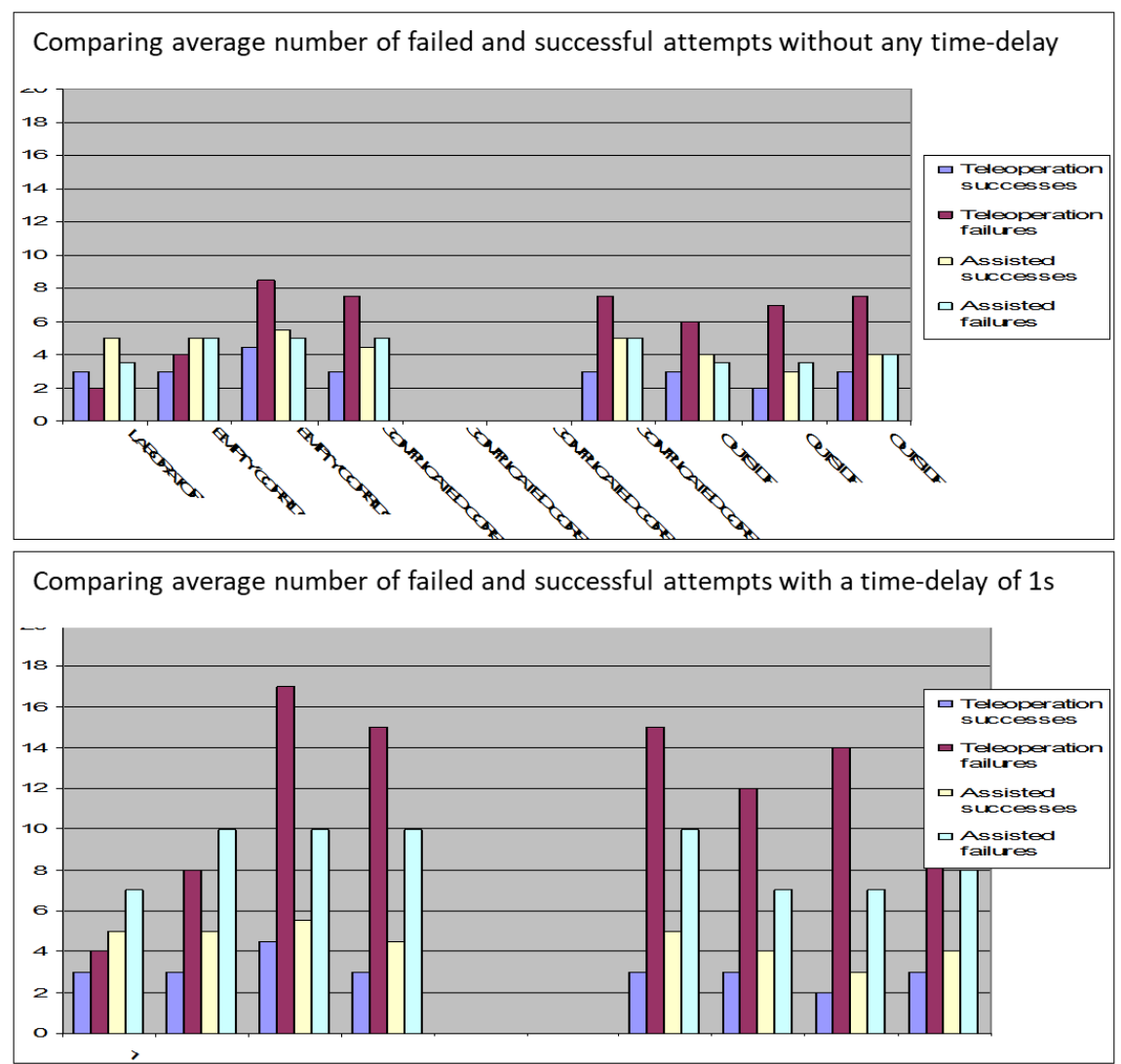

Fig. 6. Comparing average number of failed and successful attempts with 1s delay

\section{Discussion and Conclusions}

In simpler situations, wheelchair drivers performed faster without sensors assisting them but in more complicated environments or with longer time delays then they were quicker with sensors assisting them. With wider gaps or in simpler situations then drivers consistently performed faster without help. As gaps reduced or environments became more complicated, or as time delays increased then drivers found driving more difficult and the sensors became more and more useful. When the situations became more complicated then drivers performed better with help from the sensors. As gaps reduced, assistive systems were consistently quicker than human drivers by themselves.

Time-delays were only introduced between the joystick and controller. Delays could be introduced elsewhere. Further statistical analysis could be conducted and delay compensation. A delay could have been introduced in two places but it was only 
introduced in displaying the camera view to the tele-operator. The system needs to be retested with a delay after the joystick and before transmitting the movement instructions to the mobile robot as results may then be significantly different. In any real system a delay would probably be present in both if it was present in one.

An implication of the results is that sensors should not be used in freely navigable regions with good views but should be reserved for more complicated situations.

Intelligence [31-34], input devices [35] and force sensing [36] could be included. Modelling [37-40] and decision making [40-46] are now being investigated for future application.

\section{Acknowledgment}

Research in this paper was funded by EPSRC grant EP/S005927/1 and supported by The Chailey Heritage Foundation and the University of Portsmouth.

\section{References}

1. I.T. Draper, R. Johns, "The disordered movement in parkinsonism and the effect of drug treatment". Johns Hopkins Hosp 115, pp: 465-480, 1964.

2. D. Sanders, M. Langner, N. Bausch, Y. Huang, S.A. Khaustov, S. Simandjuntak, "Improving human-machine interaction for a powered wheelchair driver by using variable-switches and sensors that reduce wheelchair-veer". Intelligent Systems and Applications. Adv. Intell. Syst. Comput. vol. 1038, Springer, pp. 1173-1191 2019.

3. D. Sanders, O.M. Okonor, M. Langner, M. Hassan Sayed, S.A. Khaustov, P.O. Omoarebun, "Using a simple expert system to assist a powered wheelchair user". Intelligent Systems and Applications. Adv. Intell. Syst. Comput. vol. 1037, Springer, pp. 662-379, 2019.

4. D. Sanders, G. Tewkesbury, H. Parchizadeh, J.J. Robertson, P.O. Omoarebun, M. Malik, "Learning to drive with and without intelligent computer systems and sensors to assist". Adv. Intell. Syst. Comput. vol. 868, Springer, pp. 11711181, 2019.

5. D. Sanders, A. Gegov, M. Haddad, F. Ikwan, D. Wiltshire, Y.C. Tan, "A rulebased expert system to decide on direction and speed of a powered wheelchair". Intelligent Systems and Applications: vol. 1. Adv. Intell. Syst. Comput. vol. 868, Springer, pp. 822-838, 2019.

6. P. Fiorini, R. Oboe, "Internet-based telerobotics: Problems and approaches", Proc' ICAR'97, Monterey, CA), pp. 765-770. Teleoperation of robots with delay 681. 1997.

7. N Richard "Time delay systems: An overview of some recent advances and open problems", Automatica 39, pp 1667-1694. 2003. 
8. Lawrence DA. "Stability and transparency in bilateral teleoperation," IEEE Trans. Robot. Autom. 9 (5). 2003.

9. W. Kim, B. Hannaford, A. Bejczy, "Force reflection and shared compliant control in operating telemanipulators with time delay", IEEE Trans. Robot. Autom. 8(2), 176-185. 1992.

10. E. Slawinski, V. Mut, J.F. Postigo, "Teleoperation of robots with time-varying delay". Robotica (2006) volume 24, pp. 673-681. 2006.

11. J. Brumlik, B. Boshes. "The mechanism of bradykinesia in parkinsonism". Neurology (Minneap) 16:337-44. 1966.

12. M. Joubert, A. Barbeau. "Akinesia in Parkinson's disease". Progress in Neurogenetics. Excerpta Medica Foundation pp:366-376. 1969.

13. M. Wiesendanger, P. Schneider, J.P. Villoz. "Electromyographic analysis of a rapid volitional movement". Am J Physical Med 48: 17-24. 1969.

14. R.J. Kosinski, "A Literature Review on RT Kinds of RT Experiments." https://www.semanticscholar.org/paper/A-Literature-Review-on-ReactionTime-Kinds-of-Time-Kosinski. 2012.

15. D. Sanders, A. Gegov, G. Tewkesbury, R. Khusainov, "Sharing driving between a vehicle driver and a sensor system using trust-factors to set control gains". Intelligent Systems and Applications: vol 1. Adv. Intell. Syst. Comput. vol. 868, Springer, pp. 1182-1195. 2019.

16. D.A. Sanders "Comparing speed to complete progressively more difficult robot paths between human drivers and humans with sensor systems to assist". Assembly Automation. AA-08-057. 2009.

17. D.A. Sanders, S. Urwin-Wright, G.E. Tewkesbury et al. Pointer device for thin-film transistor and cathode ray tube computer screens. Electronics Letters 41 (16), pp: 894-896. 2005.

18. I.J. Stott, D.A. Sanders "New powered wheelchair systems for the rehabilitation of some severely disabled users". Int Jnl of Rehabilitation Research 23 (3), pp: 149-153. 2000.

19. D.A. Sanders "Controlling the direction of "walkie" type forklifts and pallet jacks on sloping ground". Assemb Auto, 28 (4), pp 317-324. 2008.

20. I.J. Stott, D.A. Sanders The use of virtual reality to train powered wheelchair users and test new wheelchair systems. Int Jnl of Rehabilitation Research 23 (4), pp 321-326. 2000.

21. R.J. Anderson, M. Spong Bilateral control of teleoperators with time delay," IEEE Trans. Autom. Control 34(5), pp: 494-501, 1989.

22. J.Y. Chen, E.C. Haas, M.J. Barnes, "Human Performance Issues and User Interface Design for Teleoperated Robots". IEEE Trans on Systems, Man, and Cybernetics, Part C: Apps and Rev, 37, pp 1231-1245. 2007.

23. D.A. Sanders, M. Langner, A.E. Gegov, D. Ndzi, H. Sanders, G.E. Tewkesbury, "Driver performance and their perception of system time

24. lags when completing robot tasks". Proc 9th Int Conf Human Systems Interaction. IEEE, pp. 236-242. 2016. 
25. W. Gao, M Hinders "Robot sonar backscatter algorithm for automatically distinguishing walls, fences, and hedges". Int Jnl of Robotics Research 25 (2), pp 135-145. 2006.

26. D.A. Sanders, A. Baldwin "X-by-wire technology". Total Vehicle Technology: Challenging current thinking, pp 3-12. 2001.

27. D.A. Sanders "Comparing ability to complete simple nuclear rescue or maintenance tasks with a robot for a human driver and a human with a sensor system to assist". Advanced Robotics 8011. 2009.

28. D. Sanders, "Analysis of failure rates with a robot between a human driver and a human with a sensor system to assist". Robotica. 2009.

29. D. Sanders, "Microprocessing and microprogramming", 38, p 833. 1993.

30. D.A. Sanders, "The modification of pre-planned manipulator paths to improve the gross motions associated with the pick and place task", Robotica, Vol. 13 pp.77-85. 1995.

31. G.E. Tewlesbury, D.A. Sanders "A new robot command library which includes simulation”, Ind' Robot; an int' jnl, Vol 26, No 1, pp 39-48. 1999.

32. D.A. Sanders, I.J. Stott IJ, "A new prototype intelligent mobility system to assist powered wheelchair users". Ind' Robot; an int' jnl, Vol 26, No 6, pp 466-475. 1999.

33. M. Haddad, D. Sanders, N. Bausch, G. Tewkesbury, A. Gegov, M. Hassan Sayed, "Learning to make intelligent decisions using an Expert System for the intelligent selection of either PROMETHEE II or the Analytical Hierarchy Process". Intelligent Systems and Applications: vol 1. Adv. Intell. Syst. Comput. vol. 868, Springer, pp. 1303-1316 2019.

34. D. Sanders, D.C. Robinson, M. Hassan Sayed, M.J.M. Haddad, A. Gegov, N. Ahmed, "Making decisions about saving energy in compressed air systems using Ambient Intelligence and Artificial Intelligence". Adv. Intell. Syst. Comput. vol. 869, Springer, pp. 1229-1236, 2019.

35. D. Sanders, "A pointer device for TFT display screens that determines position by detecting colours on the display using a colour sensor and an Artificial Neural Network". Displays. DISPLA-D-08-00006. 2009.

36. D.A. Sanders S. Urwin-Wright, G.E. Tewkesbury et al. "Pointer device for thin-film transistor and cathode ray tube computer screens". Electronics Letters, 41 (16), pp 894-896. 2005.

37. D. Sanders. Force sensing. Ind Rob -an int' jnl: 34 (4), pp 268-268. 2007.

38. D.A. Sanders. "Recognising shipbuilding parts using ANNs and Fourier Descripors". IMechE Part B: Jnl of Eng Manf. JEM1382. 2009.

39. D. Sanders, "Real time geometric modelling using models in an actuator space and Cartesian space", Jnl of Robotic Systems, vol. 12 No.1, pp.19-28. 1995.

40. D.A. Sanders. "Progress in Machine Intelligence". Industrial Robot - An International Journal Volume: 35 Issue: 6, Pages: 485-487. 2008.

41. D. Sanders, Q. Wang, N. Bausch, Y. Huang, S.A. Khaustov, I. Popov, “A method to produce minimal real time geometric representations of moving obstacles. Intelligent Systems and Applications: vol 1. Adv. Intell. Syst. Comput. vol. 868, Springer, pp. 881-892, 2019. 
42. M.J.M Haddad, D. Sanders, A. Gegov, M. Hassan Sayed, Y. Huang, M. AlMosawi, "Combining multiple criteria decision making with vector manipulation to decide on the direction for a powered wheelchair". Intelligent Systems and Applications. Adv. Intell. Syst. Comput. vol. 1037, Springer, pp. 680-693, 2019.

43. M.J.M Haddad, D. Sanders, N. Bausch, "Selecting a robust decision making method to evaluate employee performance", International Journal of Management and Decision Making, vol. 18, no. 4, pp. 333-351. 2019.

44. M.J.M Haddad, D. Sanders, G.E. Tewkesbury, "Selecting a discrete Multiple Criteria Decision Making method to decide on a corporate relocation", Archives of Business Research, vol. 7, no. 5, pp. 48-67. 2019.

45. M.J.M. Haddad, D. Sanders, "Selecting a best compromise direction for a powered wheelchair using PROMETHEE', IEEE Trans on Neural Systems and Rehab Engineering, vol. 27, no. 2, pp. 228-235. 2019.

46. M.J.M Haddad, D. Sanders, G. Tewkesbury, A. Gegov, M. Hassan Sayed, F.C. Ikwan, "Initial results from using Preference Ranking Organization METHods for Enrichment of Evaluations to help steer a powered wheelchair". Intelligent Systems and Applications. Adv. Intell. Syst. Comput. 1037, Springer, pp. 648661, 2019 